\title{
Magnetic dipole strength functions in heavy deformed nuclei
}

\author{
Dieter Zawischa \\ Institut für Theoretische Physik der Universität Hannover, D-3000 Hannover, Federal Republic of Germany \\ Malcolm Macfarlane and Josef Speth \\ Institut für Kernphysik der Kernforschungsanlage Jülich, Postfach 1913, D-5170 Jülich, Federal Republic of Germany \\ and Nuclear Theory Center, Indiana University, Bloomington, Indiana 47405
}

(Received 8 August 1989)

\begin{abstract}
The isovector spin-flip $M 1$ strength function in the deformed heavy nuclei ${ }^{156} \mathrm{Gd}$ and ${ }^{238} \mathrm{U}$ is calculated in the quasiparticle random-phase approximation. Interaction parameters are adjusted to reproduce the energies of isobaric-analog and Gamow-Teller excitations observed in $(p, n)$ chargeexchange reactions. A phenomenological spreading width for decay into background $2 \mathrm{p} 2 \mathrm{~h}$ states is introduced and adjusted to reproduce the $M 1$ spin-flip strength function recently observed by elastic scattering of tagged photons on ${ }^{90} \mathrm{Zr}$ and ${ }^{208} \mathrm{~Pb}$. With the model parameters fixed in this fashion, our calculations predict large quantities of spin-flip $M 1$ strength $\left(10-20 \mu_{N}^{2}\right)$ between 5 and $10 \mathrm{MeV}$ excitation in ${ }^{156} \mathrm{Gd}$ and ${ }^{238} \mathrm{U}$; it is strongly fragmented and should be observable by tagged-photon elastic scattering and proton inelastic scattering but probably not in high resolution inelastic electron scattering.
\end{abstract}

\section{INTRODUCTION}

The magnitude, location, and distribution of magnetic dipole strength in heavy nuclei were almost unknown before 1980. Theoretical studies predicted relatively strong isovector $1^{+}$excitations in nuclei wherein spin-orbit partners of large angular momentum are on opposite sides of the Fermi surface.

In the past ten years a series of pioneering experimental studies has altered this situation radically. The main components of the long-missing $M 1$ strength have now been found in at least a few heavy nuclei. The crucial experiments fall into three categories with different probes and a variety of energies.

(1) The discovery and study of the $\Delta T_{z}=-1 M 1$ spinflip or Gamow-Teller (GT) strength by Goodman and collaborators ${ }^{1}$ at the Indiana University Cyclotron Facility in $100-200 \mathrm{MeV}(p, n)$ charge-exchange reactions.

(2) The discovery and study of orbital isovector $M 1$ strength in deformed nuclei by Richter and collaborators, ${ }^{2}$ of the University of Darmstadt Linear Electron Accelerator in high-resolution studies of the inelastic scattering of 20-40 MeV electrons.

(3) Identification of widely fragmented $\Delta T_{z}=0$ spin-flip isovector $M 1$ strength by Laszewski and collaborators ${ }^{3,4}$ at the University of Illinois Electron Accelerator in the elastic scattering of 5-10 MeV polarized tagged photons.

Much experimental work remains to be done since $M 1$ strength in heavy nuclei is now known to be spread over many states and has been clearly identified in rather few nuclei. However, a consistent picture is beginning to emerge. It is in good general agreement with rather long-standing theoretical predictions. In this paper we discuss quasiparticle random-phase approximation (QRPA) calculations for spin-flip isovector $M 1$ strength in a variety of nuclei, treating both the $\Delta T_{z}=-1$
Gamow-Teller excitations and the $\Delta T_{z}=0$ inelastic excitations.

In only a very few cases is the majority of the calculated $M 1$ strength concentrated in a single state. Wellknown examples of such sharp $M 1$ states are ${ }^{12} \mathrm{C}$ (Ref. 5) and ${ }^{48} \mathrm{Ca} .{ }^{6}$ However in heavier nuclei like ${ }^{90} \mathrm{Zr}$ and ${ }^{208} \mathrm{~Pb}$ the experimental situation is less clear. Calculations in the one-particle one-hole random-phase approximation (1p1h RPA) including only nucleonic degrees of freedom predict one strong $1^{+}$state around $8-9 \mathrm{MeV}$ in ${ }^{90} \mathrm{Zr}$ with predominant configuration $v\left(g_{7 / 2} g_{9 / 2}^{-1}\right)$. In ${ }^{208} \mathrm{~Pb}$ an even stronger (isovector) $1^{+}$resonance (strength roughly three times that of the corresponding excitation in ${ }^{90} \mathrm{Zr}$ ) is predicted in the region between $7-8 \mathrm{MeV}$ with dominant configurations $\pi\left(h_{9 / 2} h_{11 / 2}^{-1}\right)$ with $v\left(i_{11 / 2}, i_{13 / 2}^{-1}\right)$. These are nearly pure spin-flip excitations and can be therefore excited by real $^{7}$ and virtual ${ }^{3,4,8}$ photons and by protons ${ }^{9}$ and neutrons. ${ }^{10}$ On the other hand, $1^{+}$transitions dominated by the convection current (orbital) contribution are excited very weakly in proton inelastic scattering. Spin-flip states are strong as predicted by $1 \mathrm{p} 1 \mathrm{~h}$ RPA calculations with much of the strength concentrated in one single level would have been detected easily in inelastic electron and proton scattering; none have yet been detected in these reactions.

In heavy nuclei, however, in the energy region where those $1^{+}$states are expected, the density of two-particle two-hole $(2 \mathrm{p} 2 \mathrm{~h})$ states is high. Therefore the $1 \mathrm{p} 1 \mathrm{~h}$ configurations couple strongly with the $2 \mathrm{p} 2 \mathrm{~h}$ configurations, fragmenting the $M 1$ strength in the expected energy region. In addition, the $M 1$ strength is further reduced by coupling to non-nucleonic degrees of freedom (admixture of $\Delta$ particle nucleon-hole configurations). Such calculations have been performed for ${ }^{90} \mathrm{Zr}$ and ${ }^{208} \mathrm{~Pb}$ some years ago by Cha et al. ${ }^{11}$ Here it turned out that these additional effects reduce the total 
$M 1$ strength below $11 \mathrm{MeV}$ by roughly a factor of 2 compared to the $1 \mathrm{p} 1 \mathrm{~h}$ RPA results. The recent experiments of Laszewski et al. ${ }^{3,4}$ with polarized tagged photons seem to support these predictions.

Information on $M 1$ strength in deformed nuclei is still sparse. Predominantly orbital $M 1$ excitations have been found by $\left(e, e^{\prime}\right)$ at the Darmstadt Linear Electron Accelerator $^{2}$ and by nuclear resonance fluorescence ${ }^{12}$ at around $3 \mathrm{MeV}$ in rare earth nuclei. The existence and location of such orbital $M 1$ strength is rather well understood. In a recent paper ${ }^{13}$ it was shown that the purity of the low energy orbital $M 1$ excitations are a consequence of the strongly repulsive residual particle-hole interaction in the spin-isospin channel. This repulsive interaction pushes the dominant spin-flip $M 1$ strength up to excitation energies of 5-10 MeV leaving the orbital components behind close to their unperturbed position. The predicted spin-flip strength between 5 and $10 \mathrm{MeV}$ for deformed nuclei has not yet been found. In the original paper the single-particle wave functions used contain an error noted and corrected in the erratum to Ref. 13. The distribution of orbital strength is only slightly influenced and the qualitative interpretation of both orbital and spin-flip modes is unaltered. Similar conclusions have been reached by Dietrich et al. ${ }^{14}$

In this paper we investigate in detail the spin-flip $M 1$ strength in deformed nuclei, focusing on ${ }^{156} \mathrm{Gd}$ and ${ }^{238} \mathrm{U}$. The connection between the various sorts of $M 1$ strength studied in $(p, n)$ charge exchange and in elastic electron and proton scattering can be seen from the structure of the (nonrelativistic) nuclear effective $M 1$ operator. The isovector part of this operator is

$$
\mathbf{M}_{\mu}^{1}=\sum_{i=1}^{A} g_{1} l_{i} t_{\mu}^{1}(i)+\frac{1}{2}\left(g_{s}^{(p)}-g_{s}^{(n)}\right)\left(\mathbf{M}_{\mathrm{GT}}\right)_{\mu}^{1},
$$

where the Gamow-Teller operator is

$$
\left(\mathbf{M}_{\mathrm{GT}}\right)_{\mu}^{1}=\sum_{i} \boldsymbol{\sigma}_{i} t_{\mu}^{1}(i)
$$

The separation into orbital and spin-flip parts is evident. The $\Delta T_{z}=\mu=-1$ isospin component is active in $(p, n)$ charge exchange. Here, although the GT excitation has three isospin components $\left(T=T_{0}-1, T_{0}, T_{0}+1\right.$ with $T_{0}$ the target isospin) $99 \%$ of the strength is in the lowest- $T$ $\left(T_{0}-1\right)$ component. The $T_{0}$ component is excited in elastic electron or proton scattering. Thus the $\Delta T_{z}=0$ spin-flip strength seen in inelastic scattering is an isospin component of the GT excitation not accessible in $(p, n)$ charge exchange. We note also that the $\Delta T_{z}=0$ orbital part of the isovector $M 1$ operator is

$$
\left(\mathbf{M}_{0}^{1}\right)_{\text {orbital }}=\frac{1}{2}\left(\mathbf{L}_{p}-\mathbf{L}_{n}\right),
$$

a generator of orbital rotations of neutrons against protons. However, the strength of the orbital excitation is much too small to permit any collective scissor-mode interpretation.

The paper is organized as followed. We first introduce the model and apply it in Sec. III to GT resonances in ${ }^{156} \mathrm{Gd},{ }^{165} \mathrm{Ho}$, and ${ }^{238} \mathrm{U}$. A comparison with the experimental data strongly constrains the magnitude of the re- sidual interaction in the spin-isospin channel. In Sec. IV we give the results of the model for the $\Delta T_{z}=0$ spin-flip $M 1$ strength distribution. These results will be discussed in the light of experimental and theoretical knowledge of the corresponding spin-flip strength in ${ }^{90} \mathrm{Zr}$ and ${ }^{209} \mathrm{~Pb}$. Finally, in our summary, we argue that a consistent theoretical picture of $M 1$ strength in nuclei is emerging, based on the mean-field description of nuclei and on well-established properties of the residual interaction.

\section{COMMENTS ON THE QUASIPARTICLE RANDOM-PHASE APPROXIMATION (QRPA)}

The calculations of the $M 1$ and GT strength functions have been performed in the QRPA. This successful model of the nuclear many-body system is based on the mean-field approximation. In this connection, the deformed rare earth and actinide nuclei are of special interest because they are well described by the unified model, in which independent particles in a deformed mean field rotate adiabatically. For the deformed intrinsic wave function, the total angular momentum $j$ is no longer a good quantum number, only its projection $K$ on the symmetry axis, and the parity. The selection rules for $M 1$ transitions are $\Delta K^{\pi}=1^{+}$or $\Delta K^{\pi}=0^{+}$. In QRPA, the state vectors of excited states consist of two parts: the first one is a superposition of two-quasiparticle excitations built upon a correlated ground state, the other one results from the destruction of two quasiparticles in a virtual four-quasiparticle ground-state fluctuation:

$$
|m\rangle=\frac{1}{2} \sum_{\lambda, \mu}\left(X_{\lambda \mu}^{m} \alpha_{\lambda}^{\dagger} \alpha_{\bar{\mu}}^{\dagger}+Y_{\lambda, \mu}^{m} \alpha_{\bar{\lambda}} \alpha_{\mu}\right)|0\rangle
$$

where the $\alpha_{\lambda}^{\dagger}$ and $\alpha_{\mu}$ are Bogoliubov quasiparticle operators and the overbar on the indices indicates the timereversed single-particle state. The amplitudes $X^{m}$ and $Y^{m}$ as well as the excitation energies, are solutions of the QRPA. All the calculations have been performed in the manner described in Ref. 15. The single-particle wave functions have been generated from a nonspherical Woods-Saxon potential, as described in Ref. 15 .

The particle-hole interaction is of the Landau-Migdal type

$$
\begin{aligned}
F^{\mathrm{ph}=}=C_{0} \delta\left(\mathbf{r}_{1}-\mathbf{r}_{2}\right) & \left(f_{0}+f_{0}^{\prime} \boldsymbol{\tau}_{1} \cdot \tau_{2}+g_{0} \boldsymbol{\sigma}_{1} \cdot \boldsymbol{\sigma}_{2}\right. \\
& \left.+g_{0}^{\prime} \boldsymbol{\sigma}_{1} \cdot \boldsymbol{\sigma}_{2} \boldsymbol{\tau}_{1} \cdot \tau_{2}\right)+F^{\text {vel dep }} .
\end{aligned}
$$

The velocity dependent part $F^{\text {vel dep }}$ turns out to be of little importance in the present context, although it has some influence on the orbital $M 1$ strength. It will be discussed no further here. The same parameters have been used as in Ref. 13 except for the spin-isospin flip strength $g_{0}^{\prime}$ which will be adjusted to reproduce the GT resonances. The components of the spurious (isoscalar) $1^{+}$ state are projected out in the manner described in Ref. 13.

The isovector part of the magnetic dipole operator 
[e.g., (1.1)] can be written as

$$
\mathbf{M}_{\mu}^{1}=\sum_{i=1}^{A}\left[g_{l}^{(i)} \mathbf{j}_{l} t_{\mu}^{1}(i)+\frac{1}{2}\left(g_{s}^{(p)}-g_{s}^{(n)}-2 g_{l}^{(l)}\right) \boldsymbol{\sigma}_{i} t_{\mu}^{1}(i)\right]
$$

Equation (2.3) shows that transitions due to the orbital part of the operator are possible between states of the same $j$ shell, so that no orbital excitations are expected in spherical closed shell nuclei only. On the other hand, magnetic spin-flip transitions between spin-orbit partners are possible. In deformed nuclei, the Fermi surface generally separates Nilsson orbitals originating from the same $j$ shell; such states are connected by large matrix elements of the operator $\mathbf{J}$. This is the origin of the lowenergy orbital excitations in nonspherical nuclei. ${ }^{13,16,17}$ In the present calculation we concentrate on the spin-flip transitions between spin-orbit partners.

The truncation of a configuration space gives rise to renormalized effective interactions and to renormalized effective transition operators. In some cases conservation laws determine the form of the effective operators. ${ }^{18}$ This is not the case for the $M 1$ operator in, e.g., (2.3). For magnetic moments and transitions in odd-mass nuclei an effective operator has been determined that accounts for correlations beyond the $1 \mathrm{p} 1 \mathrm{~h}$ RPA configuration space. ${ }^{19}$ The main effect of effective-operator modifications can be included in the QRPA calculations by using effective $g_{l}$ and $g_{s}$ parameters in Eq. (2.3) instead of those appropriate for free nucleons. In the following, the effective $M 1$ operator is used with modified $g_{s}$ values only:

$$
\begin{aligned}
& g_{l}^{p(\mathrm{eff})}=g_{l}^{p=1}, \\
& g_{l}^{n(\mathrm{eff})}=g_{l}^{n}=0, \\
& g_{s}^{(\mathrm{eff})}=0.7 g_{s} .
\end{aligned}
$$

For all further details we refer to Refs. 13 and 15.

\section{GAMOW-TELLER RESONANCES}

In medium and heavy nuclei the Gamow-Teller resonances (GTR) dominate the $0^{\circ}(p, n)$ spectrum induced by medium-energy protons $(100 \mathrm{MeV} \leq E p \leq 400 \mathrm{MeV})$. These $1^{+}, \Delta L=0, \Delta S=1$ resonances have been extensively studied experimentally (e.g., Ref. 19) and theoretically. The GTR lie close to the $0^{+}, \Delta L=0, \Delta S=0$ isobaric ana-
TABLE I. Landau parameters used in the present QRPA calculation. The values in parentheses have been used in Ref. 13.

\begin{tabular}{ll}
\hline \hline$f_{0}^{\text {in }}=0.21$ & $f_{0}^{\mathrm{ex}}=-2.45$ \\
$f_{0}^{\prime \text {,In }}=0.585(0.56)$ & $f_{0}^{\prime \mathrm{ex}}=1.71(1.65)$ \\
$g_{0}^{\mathrm{\prime n}}=g_{0}^{\mathrm{ex}}=0.1$ & $g_{0}^{\prime \mathrm{n}}=g_{0}^{\prime \mathrm{ex}}=0.7$ \\
$f_{1}=-0.7$ & $f_{1}^{\prime}=0.35$ \\
& \\
\hline
\end{tabular}

$\log$ resonances (IAR) detected much earlier in low-energy $(p, n)$ reactions. The excitation energies of the two resonances coincide in ${ }^{208} \mathrm{~Pb}$. In nuclei lighter than ${ }^{208} \mathrm{~Pb}$ the GTR is slightly above the IAR, whereas in nuclei heavier than ${ }^{208} \mathrm{~Pb}$ the IAR tends to be slightly higher. These experimental facts will be used in the following to calibrate the interaction in the spin-isospin channel. This is crucial for the location of the spin-flip $M 1$ distribution. In addition, it provides us with a good test of the interaction in the spin-independent isovector channel, which is important for the low-lying orbital $M 1$ strength.

In a conventional RPA framework we describe the charge-exchange $(p, n)$ excitations as superpositions of proton particle-neutron hole pairs built on the ground state of the original (parent) nucleus. ${ }^{21}$ Within the QRPA we have to replace the particle-hole pairs of the conventional RPA by a proton and neutron quasiparticle pair, as described in the previous section. Due to the selection rules in charge exchange reactions, only the isospin-dependent part of the particle-hole interaction [Eq. (2.2)] enters. For the IAR, which are $J^{\pi}=0^{+}$excitations, the spin-dependent part does not contribute, so the location of analog resonances is determined by the magnitude of the Landau-Migdal parameter $f_{0}^{\prime}$. (Here we have always to bear in mind, that by definition, the Landau-Migdal interaction has only a direct part.) The interaction parameters $f_{0}^{\prime \prime n}$ and $f_{0}^{\prime \text { ex }}$ given in parentheses in Table I have been used in the previous calculation of the orbital dipole states. ${ }^{13}$ The theoretical excitation energies of the IAR in ${ }^{156} \mathrm{Gd},{ }^{165} \mathrm{Ho}$, and ${ }^{238} \mathrm{U}$ obtained with these parameters are also shown in parentheses in Table II where they can be compared with the corresponding experimental values. The agreement is quite good but the theoretical values are systematically too low by a few hundred $\mathrm{keV}$. For that reason we increase $f_{0}^{\prime}$ parameters by a few percent in order to obtain the optimal interac-

\begin{tabular}{|c|c|c|c|c|}
\hline \multicolumn{5}{|c|}{ Experimental and theoretical excitation energy of the IAS and GTR } \\
\hline \multirow{3}{*}{${ }^{156} \mathrm{Gd}$} & IAS & 15.9 & $(15.6)$ & 16.2 (natural Gd) \\
\hline & GTR & 17.2 & & 17.5 (interpolation between ${ }^{144} \mathrm{Sm}$ and ${ }^{165} \mathrm{Ho}$ ) \\
\hline & IAS & 16.9 & $(16.5)$ & 16.6 \\
\hline \multirow[t]{2}{*}{${ }^{165} \mathrm{Ho}$} & & a & & \\
\hline & GTR & 17.9 & & 17.8 (estimated from Fig. 5) \\
\hline \multirow{2}{*}{${ }^{238} \mathrm{U}$} & IAS & 19.7 & $(19.4)$ & $19.8 \pm 0.1$ \\
\hline & GTR & 19.6 & & Slightly below the IAR (Ref. 23) \\
\hline
\end{tabular}

TABLE II. The theoretical values have been calculated within the QRPA, using the parameters given in Table I. For comparison we give in parentheses the excitation energies of the IAS, where the "old" $f_{0}^{\prime}$ parameters (given in parentheses in Table I) have been used.

${ }^{a}{ }^{164} \mathrm{Dy}$. 
tion in the isospin channel which strongly influences the low-lying $M 1$ spectrum. The results obtained for these low-lying $1^{+}$states with the new $f_{0}^{\prime}$ parameters differ only slightly from those obtained before. ${ }^{13}$

In the case of the GTR, only the spin-isospin part $g_{0}^{\prime}$ of the residual interaction [Eq. (2.2)] contributes. We use this fact to determine the value of $g_{0}^{\prime}$. The Landau parameters depend to some extent on the single-particle model chosen and on the size of the configuration space; small changes of the order of $10-20 \%$ are possible. Such fine tuning does not contradict the basic assumption that these parameters are universal. If the model and the configuration space, however, is fixed, then the parameters must be the same for all nuclear properties calculated. (This is different, e.g., for the multipole-multipole force, where for each multipolarity new parameters have to be introduced.) In Table II we compare the calculated excitation energies of the GTR with experiment. The agreement is good and the value of $g_{0}^{\prime}=0.7$ is in the range of other determinations. From Table II it is also clear that our theoretical model can reproduce the relative energies of the IAR and GTR.

In Figs. 1-3 we show the calculated GT strength (full line) and IAR (dashed line) in ${ }^{156} \mathrm{Gd},{ }^{164} \mathrm{Dy}$, and ${ }^{238} \mathrm{U}$. The QRPA equations have been solved with the new force parameters given in Table I. The parameters are the same for all three nuclei. Experimentally, in the rare earth region, the IAR is slightly below the GTR (whereas) in ${ }^{238} \mathrm{U}$ the IAR is slightly above the GTR. ${ }^{23}$ Our theoretical results reproduce this behavior. For a more detailed comparison we show the strength distribution in ${ }^{238} U$ with an enlarged energy scale in Fig. 4. An experimental $(p, n)$ spectrum of ${ }^{165} \mathrm{Ho}$ using protons of $E_{p}=160 \mathrm{MeV}$ is shown in Fig. 5 which might be compared with our theoretical result for ${ }^{164} \mathrm{Dy}$. The narrow peak at $16.6 \mathrm{MeV}$ is the IAR which is simultaneously ex-

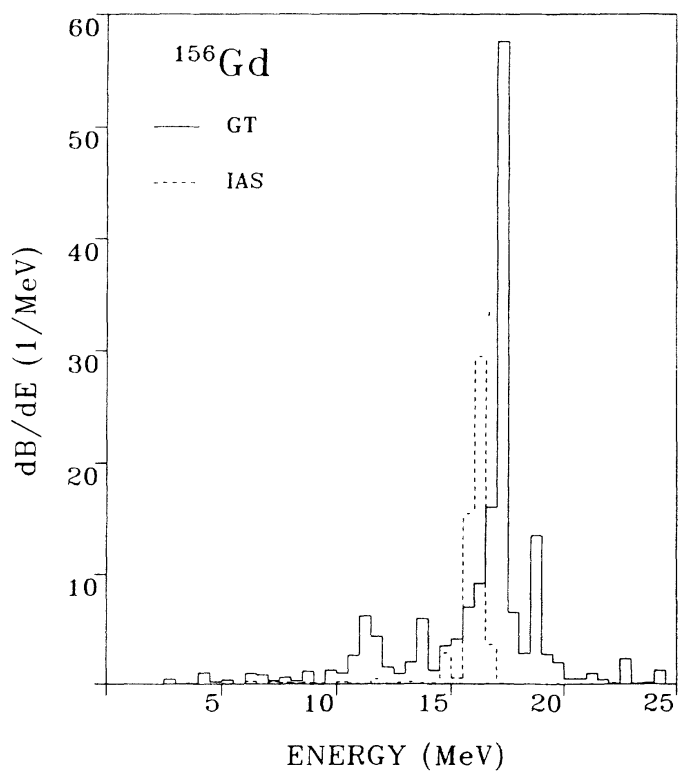

FIG. 1. Strength distributions of the Gamow-Teller resonance (solid line) and the isobaric analog state (dashed line) in ${ }^{156} \mathrm{Gd}$, calculated within the framework of the QRPA.

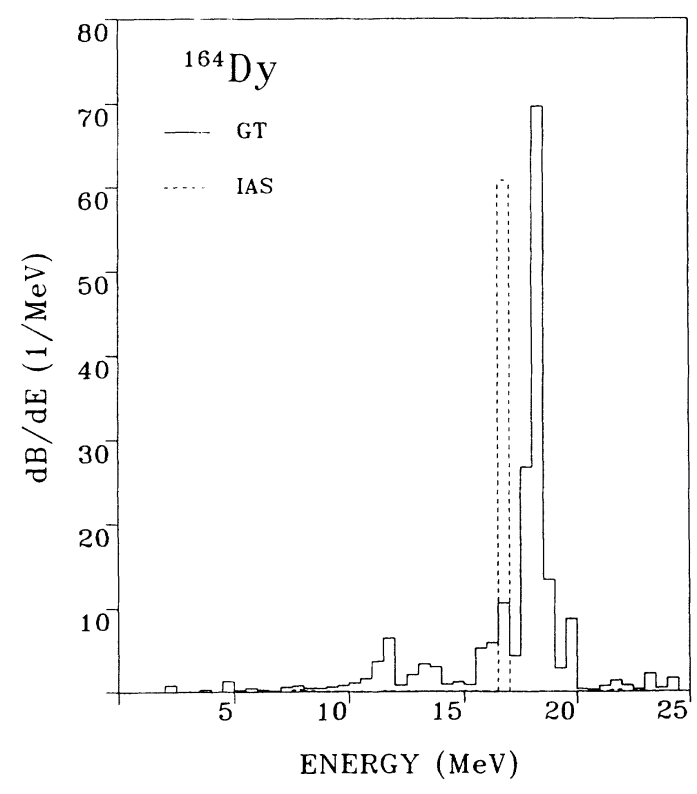

FIG. 2. Same as in Fig. 1 for ${ }^{164} \mathrm{Dy}$.

cited with the GTR. The experimental GTR energies given in Table II are estimated centroid energies of the resonance part of the GT strength. The magnitude of the GT strength in the resonance region is of the order of $55 \%{ }^{20}$ of the Ikeda sum rule, whereas our calculated strength is close to the sum-rule limit; furthermore the theoretical shape of the GTR does not agree with the experimental cross section. Here we point out that within our present model we cannot account either for the experimentally observed $50 \%$ quenching of the GT strength in the resonance region nor the width and the

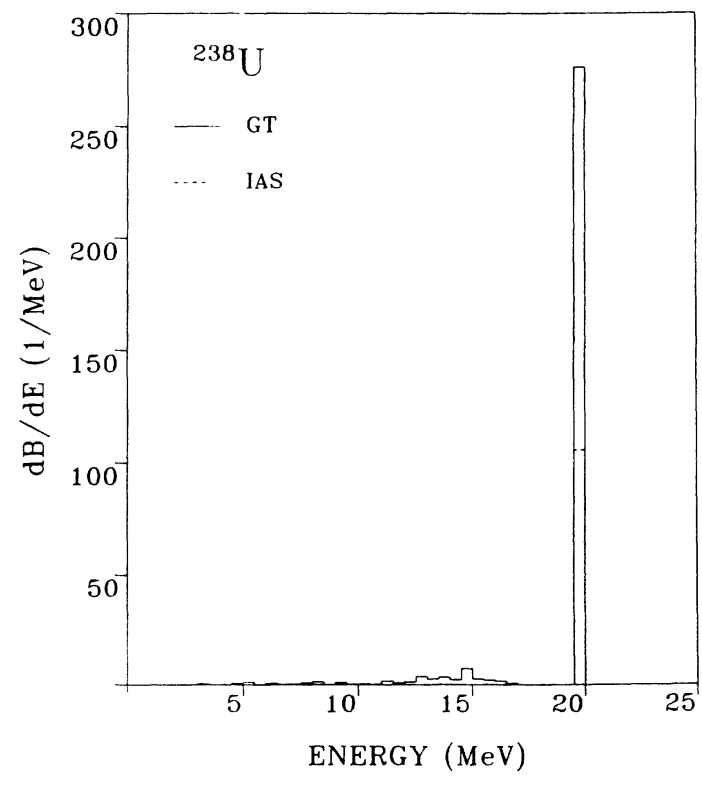

FIG. 3. Same as in Fig. 1 for ${ }^{238} \mathrm{U}$. 


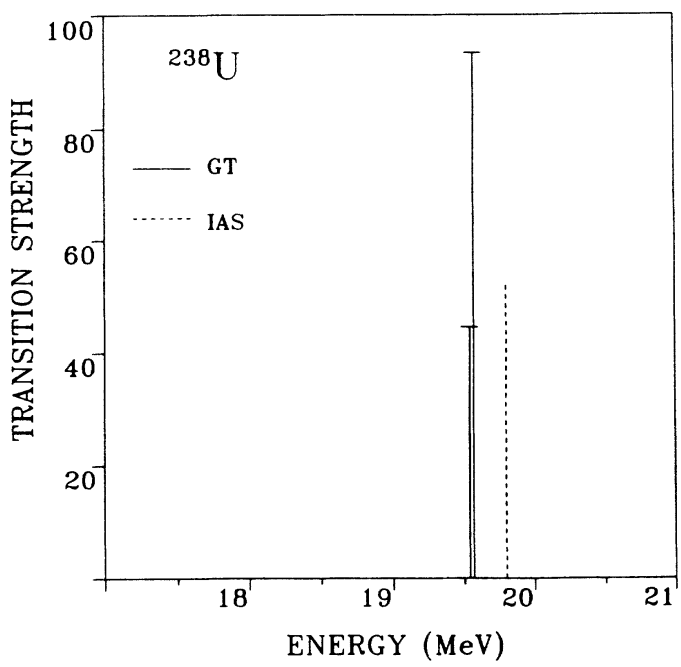

FIG. 4. The strongest Gamow-Teller (solid line) and isobaric analog (dashed line) states in ${ }^{238} \mathrm{U}$, given in an enlarged energy scale.

high-energy tail of the GTR. These limitations are well known from similar investigations in spherical nuclei. The 1p1h RPA results reported here overestimate the total strength in the resonance region by roughly a factor of 2 and the theoretical width is much smaller than the experimental one. To overcome this difficulty, not only $1 \mathrm{p} 1 \mathrm{~h}$ but also $2 \mathrm{p} 2 \mathrm{~h}$ and $\Delta$ particle-nucleon hole configurations must be included. ${ }^{11}$ Such extended models are indeed able to explain the main features of the GTR in spherical nuclei. We shall come back to this point in the next section in connection with the effective $g$ factors in the $M 1$ operator. Inclusion of $2 \mathrm{p} 2 \mathrm{~h}$ and $\Delta-h$ components in complete RPA calculations for deformed nuclei leads to very large configuration spaces and have not yet been carried out.

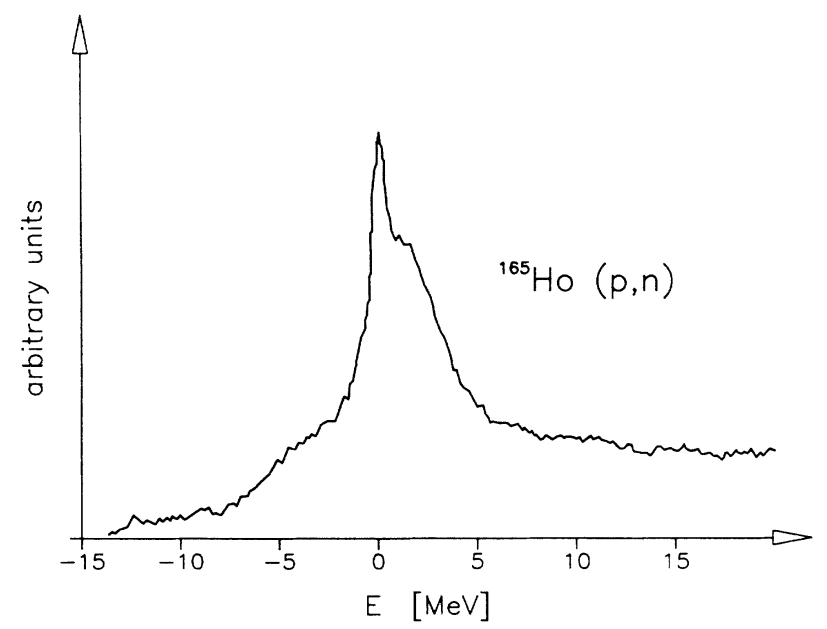

FIG. 5. Zero degree $(p, n)$ spectrum (Ref. 20) of ${ }^{165}$ Ho using protons with an incident energy $E_{p}=160 \mathrm{MeV}$. The energies are given relative to the excitation energy of the isobaric analog state $\left(E_{\mathrm{ex}}=16.6 \mathrm{MeV}\right)$.

\section{SPIN-FLIP $\Delta T_{z}=0 M 1$ STRENGTH}

The search for the spin-flip $\Delta T_{z}=0 M 1$ strength in heavy nuclei has a long history. In the early experimental work $E 1$ transitions were frequently misinterpreted as $M 1$. These (erroneous) results were in apparent agreement with theoretical predictions from $1 \mathrm{p} 1 \mathrm{~h}$ calculations, which usually give one or two strong $M 1$ states (see, e.g., Ref. 21 and references therein). With improved and more varied experments using $(n, \gamma),{ }^{10}$ nuclear-resonancefluorescence, ${ }^{8}$ high resolution $\left(e, e^{\prime}\right),{ }^{7}$ and polarized tagged photons ${ }^{3,4}$ spin-flip $M 1$ strength has been identified in a few heavy mass nuclei ${ }^{90} \mathrm{Zr},{ }^{120} \mathrm{Sn}$, and ${ }^{208} \mathrm{~Pb}$.

The spin-flip $M 1$ strength is highly fragmented and quenched by more than $50 \%$ relative to the predictions of the independent particle model (IPM). At the same time, theoretical models have made much more realistic by inclusion of the coupling to $2 \mathrm{p} 2 \mathrm{~h}-$ or rather $\mathrm{ph}+$ phonon configurations, ${ }^{24}$ or to $2 \mathrm{p} 2 \mathrm{~h}$ and $1 \Delta 1 \mathrm{~h}$ configurations. ${ }^{11}$ On this level theory and experiment are in basic agreement, as will be shown later.

There is as yet no experimental indication of the corresponding spin-flip $M 1$ strength in deformed heavy nuclei. We now give the predictions of our model for the location of the bulk of the predicted $M 1$ strength and discuss why it may have escaped detection. The calculations were performed in the framework of the QRPA as outlined in Sec. II and in previous publications. ${ }^{13,15}$ The crucial input is the ph interaction in the spin-isospin channel that has been fine tuned to reproduce the energies of $\Delta T_{z}=-1 \mathrm{GT}$ resonances, as outlined in the previous section. We have carried out detailed calculations of ${ }^{156} \mathrm{Gd}$ and ${ }^{238} \mathrm{U}$ because they have been subjected to extensive experimental study by inelastic electron scattering and they are well deformed, so that all the assumptions of our theoretical model are fulfilled. We first compare the $M 1$ strength in ${ }^{156} \mathrm{Gd}$ with the corresponding theoretical results in ${ }^{90} \mathrm{Zr}$ and ${ }^{208} \mathrm{~Pb}$. We find several similarities but also some major differences which may explain why this $M 1$ strength in the deformed nuclei has escaped experimental detection.

In Fig. 6 we show the $M 1$ strength distribution of the uncorrelated two-quasiparticle excitations in ${ }^{156} \mathrm{Gd}$ and in Fig. 7 the corresponding QRPA results. The state below $4 \mathrm{MeV}$ contains a large fraction of orbital strength as can be seen from comparing the upper and middle parts of these figures. In the upper part the full (orbital plus sign) matrix elements have been used, while in the middle part the spin $g$ factors have been put equal to zero, i.e., only the orbital contribution is plotted. For some of the strongest low-energy states of Fig. 6 which are labeled (a) to (f), the asymptotic Nilsson quantum numbers and (in parentheses) the corresponding quantum numbers in the limit of no deformation (i.e., the spherical case) are given in the caption. Practically all the low-lying twoquasiparticle states with large orbital $M 1$ strength are, in the limit $\delta \rightarrow 0$, diagonal in the quantum numbers of the spherical shell model, that is, in the total angular momentum $j$. These matrix elements have a large orbital contribution if $j$ is large. On the other hand, the higher twoquasiparticle excitations reduce in the spherical limit to 
excitations of pairs of spin-orbit partners, the matrix elements between which are purely spin-flip. The deformed single particle wave functions can be represented as linear combinations of spherical ones. For moderate deformations $0.2 \leq \delta \leq 0.3$ the asymptotic spherical state remains the dominant configuration. The unperturbed twoquasiparticle energies of the asymptotically diagonal states are directly proportional to the deformation $\delta$ (plus the BCS gap energy) and are, therefore, lower than the spin-flip transitions whose energy in the spherical case is determined by the (one-body) spin-orbit potential.

Figures 6 and 7 indicate that in deformed nuclei two different classes of magnetic excitations exist. The twoquasiparticle states up to about $4 \mathrm{MeV}$ have large orbital contributions whereas the higher two-quasiparticle excitations are predominantly of the spin-flip type. This behavior is not peculiar to ${ }^{156} \mathrm{Gd}$ but is a general feature of all well-deformed nuclei in the rare earth and actinide region. Our interest now is in the spin-flip strength; we therefore concentrate from now on the spectrum beyond $4 \mathrm{MeV}$. It is obvious that the low-lying diagonal orbital states do not exist in spherical closed shell nuclei because the two-quasiparticle states which differ only in the

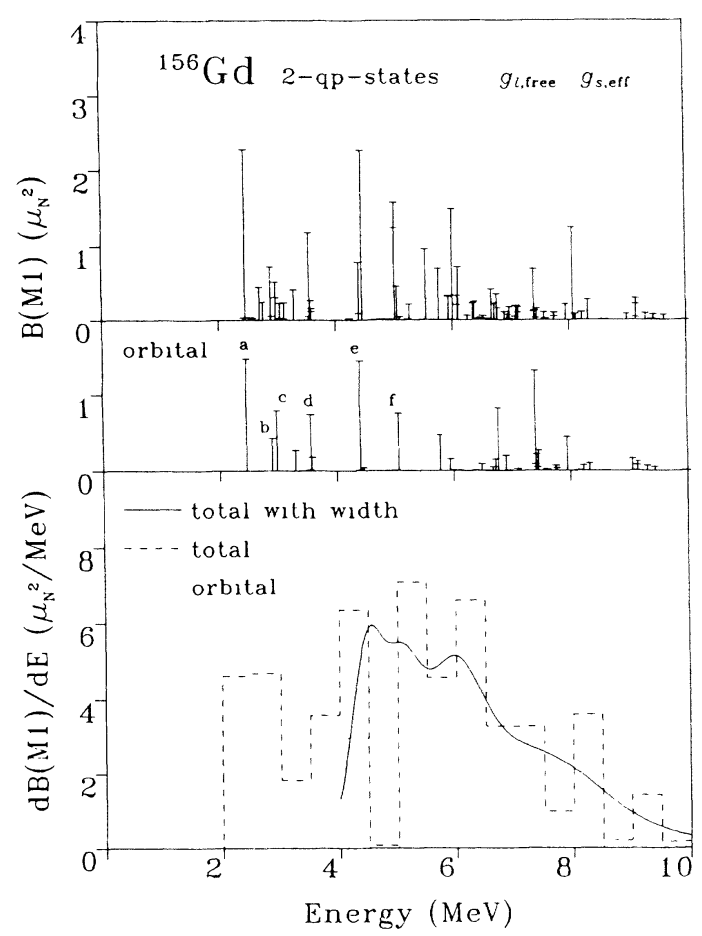

FIG. 6. $B(M 1) \uparrow$ strength distribution of the uncorrelated two-quasiparticle states in ${ }^{156} \mathrm{Gd}$. In the upper part the total strength is given, in the middle part only the orbital contribution. The quantum numbers of the more prominent low-energy orbital excitations are (a) $\frac{7}{2}^{-}\left(h_{11 / 2}\right)$ [523]- $\frac{5}{2}^{-}\left(h_{11 / 2}\right)$ [532]; (b) $\frac{5}{2}^{-}\left(h_{11 / 2}\right)[532]-\frac{3}{2}^{-}\left(h_{11 / 2}\right)[541] ;(\mathrm{c}) \frac{7}{2}^{+}\left(g_{7 / 2}\right)[404]-\frac{5}{2}^{+}\left(g_{7 / 2}\right)$ [413]; (d) $\frac{3}{2}^{+}\left(d_{5 / 2}\right) \quad[411]-\frac{1}{2}^{+}\left(d_{5 / 2}\right) \quad$ [420]; (e) $\frac{1}{2}^{+}\left(d_{3 / 2}\right)$ [411]- $-\left(-\frac{1}{2}\right)^{+}\left(d_{5 / 2}\right)$ [420]; (f) $\frac{5}{2}^{+}\left(g_{7 / 2}\right)[413]-\frac{3}{2}^{+}\left(g_{7 / 2}\right)$ [422]. In the bottom of the figure the same data are presented summed up in intervals of $0.5 \mathrm{MeV}$, and in addition with an artificial width folded in, as discussed in the text. In this figure the spurious rotational contributions have not been removed.

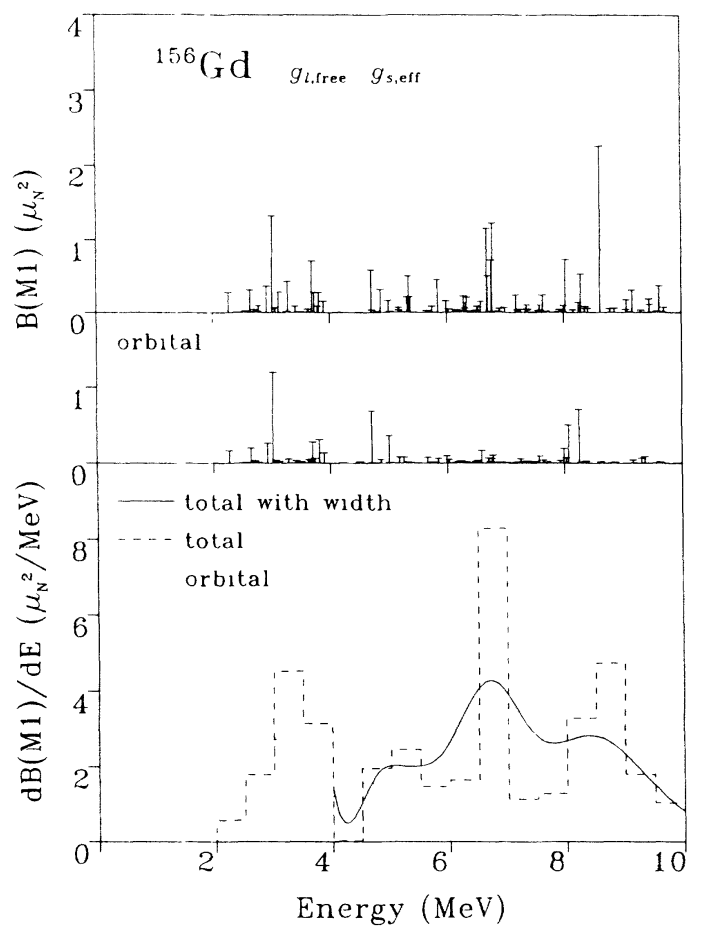

FIG. 7. Results of a QRPA calculation of the $B(M 1) \uparrow$ strength distribution. The parameters of Table I, free charges and effective $g$ factors ( $g_{\text {s eff }}=0.7 g_{\text {s free }}$ ) have been used. Compared to Ref. 13 the configuration space has been increased so that we have now included all large spin-flip transitions up to 10 $\mathrm{MeV}$. The figure is organized in the same way as Fig. 6 . In the lower part the data are presented summed up in intervals of 0.5 $\mathrm{MeV}$ (and divided by $0.5 \mathrm{MeV}$ ), and finally (above $4 \mathrm{MeV}$ ) with Gaussian functions folded into each individual state. The width of these Gaussians increases linearly from $\Gamma=0.5 \mathrm{MeV}$ at $E_{\mathrm{ex}}=4 \mathrm{MeV}$ to $\Gamma=2 \mathrm{MeV}$ at $E_{\mathrm{ex}}=10 \mathrm{MeV}$. All the states shown have been orthogonalized to the spurious rotational state so that any remnants of spuriosity are removed.

asymptotic Nilsson quantum number coalescence in the same single particle state $\left|J^{\pi}\right\rangle$ for $\delta=0$. In this limit therefore, only the spin-flip transitions remain.

The individual excitations below $4 \mathrm{MeV}$ can be compared with experiment, but for higher-excitation energy the distribution of the excitation strength is more easily appreciated if it is summed up in certain intervals. Therefore in the lower part of Figs. 6 and 7, the same data are presented once more in the form of a histogram. Finally, for the higher excitations, Gaussians with energy-dependent width have been folded in to simulate spreading effects not yet included as discussed below.

The two-quasiparticle strength function in ${ }^{156} \mathrm{Gd}$ is compared in Fig. 8 with the corresponding IPM distribution in ${ }^{90} \mathrm{Zr}$ and ${ }^{208} \mathrm{~Pb}$. The ${ }^{90} \mathrm{Zr}$ state has the configuration $v\left(g_{7 / 2} g_{9 / 2}^{-1}\right)$ and the two states in ${ }^{208} \mathrm{~Pb}$ are the well known $\pi\left(h_{9 / 2} h_{11 / 2}^{-1}\right)$ and $v\left(i_{11 / 2}, i_{13 / 2}^{-1}\right)$ spin-orbit partners. It is important to realize that the total strength in the two spherical nuclei is concentrated in one and two states, respectively, while in the deformed nucleus the strength is fragmented into many levels because the single-particle states are split by the deformation. The 
total two-quasiparticle $M 1$ strength cannot be directly compared with the $M 1$ strength in the two other nuclei because it also contains components of the "spurious" $K^{\pi}=1^{+}$state which appears because rotational symmetry has been violated by the deformed single-particle potential. Within the QRPA procedure the spurious components are projected out ${ }^{13}$ and do not appear in the
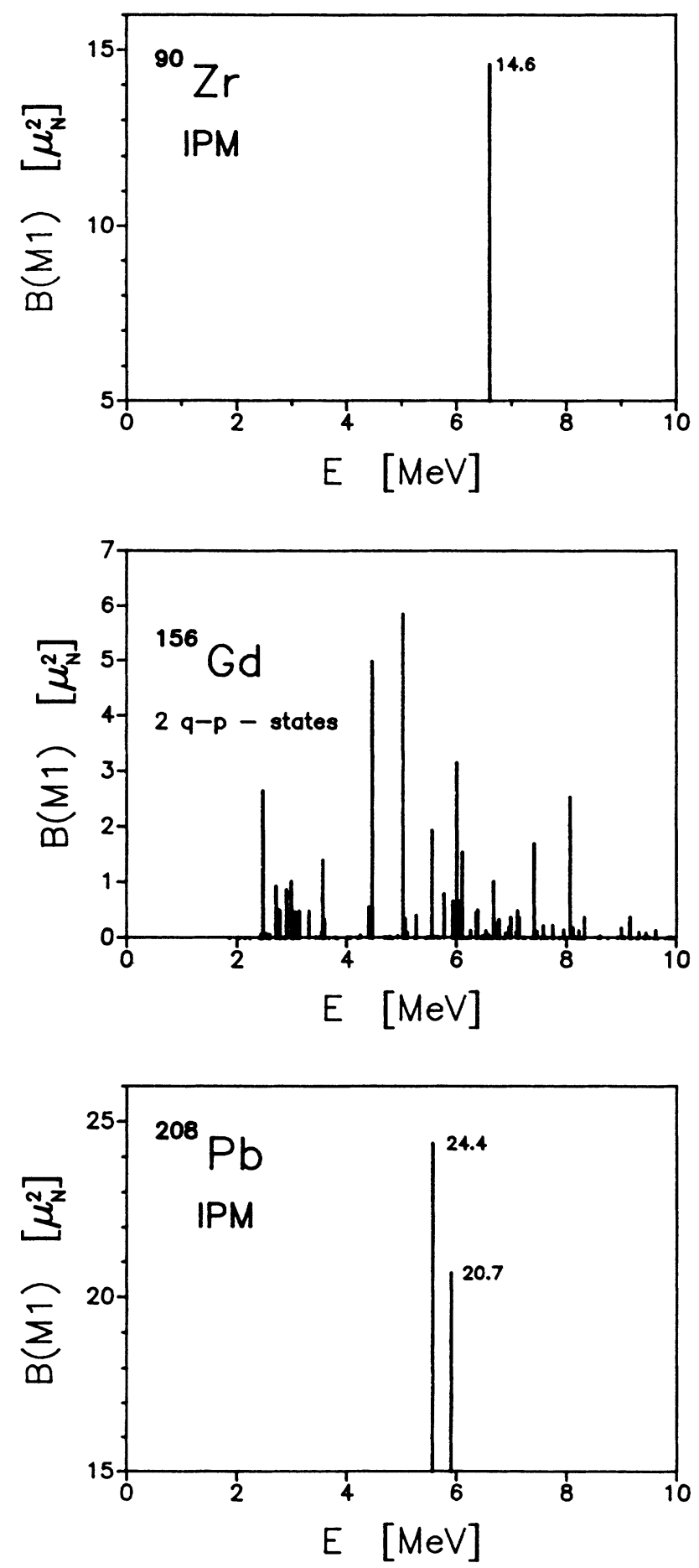

FIG. 8. $B(M 1) \uparrow$ strength distributions of the independent particle model in ${ }^{90} \mathrm{Zr}$ and ${ }^{208} \mathrm{~Pb}$ and of the uncorrelated twoquasiparticle states in ${ }^{156} \mathrm{Gd}$, respectively. The free $g$ factors have been used. Note the different scales.
QRPA spectrum.

In the middle part of Fig. 9 the $M 1$ distribution of ${ }^{156} \mathrm{Gd}$, calculated in the QRPA model (with bare operators) is shown. It is compared with the corresponding RPA results (full lines) in ${ }^{90} \mathrm{Zr}$ and ${ }^{208} \mathrm{~Pb}$. The strong
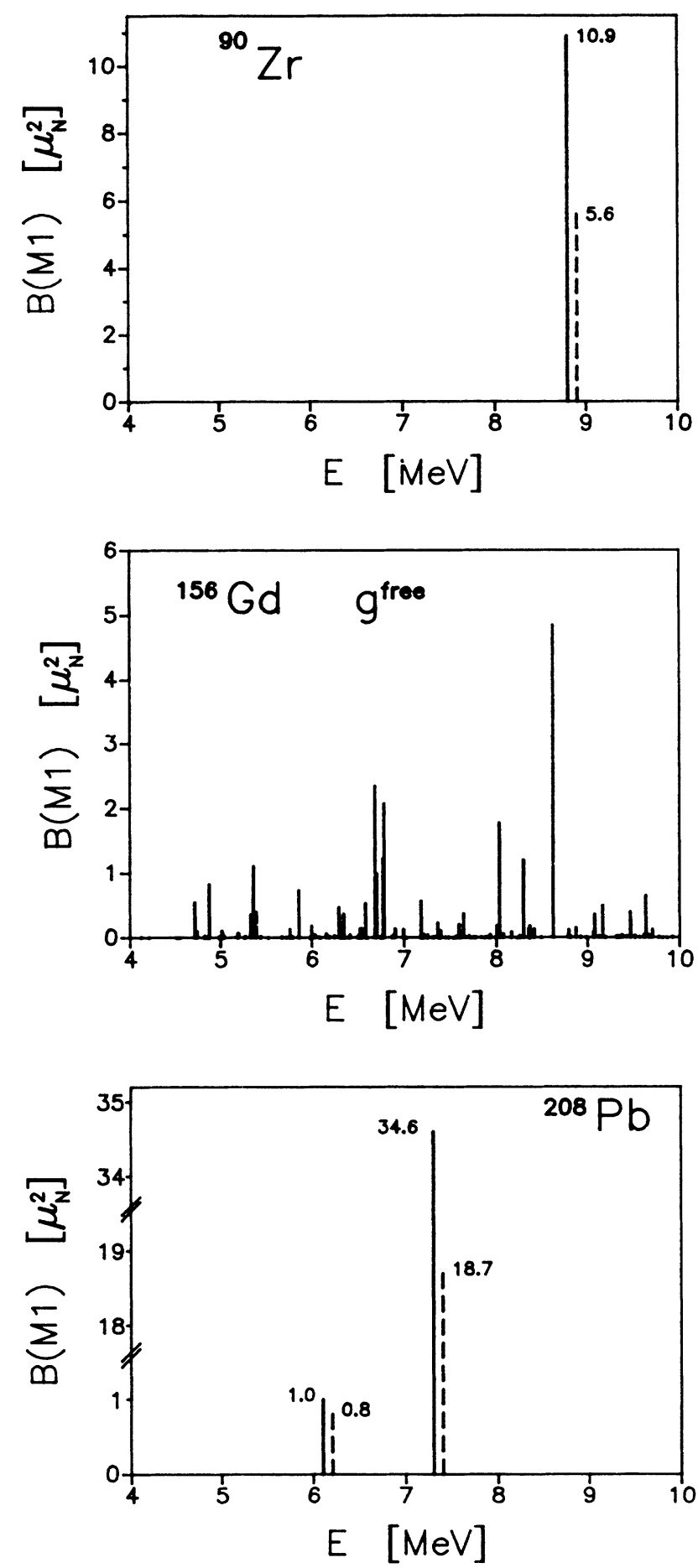

FIG. 9. The solid lines in the upper and lower parts of the figure indicate the RPA results of the $B(M 1) \uparrow$ strength distributions obtained with free $g$ factors whereas the dashed lines denote the results obtained with effective $g$ factors. In the middle part we show the QRPA results with free $g$ factors. For the results with effective $g$ factors see Fig. 7 . Note the different scales. 
repulsive ph interaction in the spin-isospin channel shifts the spin-flip strength to higher energies compared to the unperturbed results shown in Fig. 8, and reduces it through the effect of ground-state correlations. This can be seen clearly in ${ }^{90} \mathrm{Zr}$. In ${ }^{208} \mathrm{~Pb}$ the isovector part of the spin-dependent interaction produces one strong isovector state which carries nearly all the strength, whereas the small isoscalar strength is only slightly shifted because the isoscalar force $\left(g_{0}\right)$ is weak. The QRPA result in ${ }^{156} \mathrm{Gd}$ looks different. First of all there is also a shift of strength to higher energies compared to the twoquasiparticle result, again due to the strongly repulsive interaction in the spin-isospin channel. However, the $M 1$ strength is still strongly fragmented because the twoquasiparticle $M 1$ strength function is itself so fragmented that the residual interaction is unable to concentrate a major part of the $M 1$ strength in one single state. Therefore on the $1 \mathrm{p} 1 \mathrm{~h}$ RPA level, the strongest $M 1$ state in ${ }^{156} \mathrm{Gd}$ is more than a factor of 10 weaker than the isovector $M 1$ resonance in ${ }^{208} \mathrm{~Pb}$. Modification of the $M 1$ operator, as we shall see later, makes no qualitative difference; it only reduces the strength by a factor of about 2. The conclusion at this stage is that the $1 \mathrm{p} 1 \mathrm{~h}$ RPA results in ${ }^{90} \mathrm{Zr}$ and ${ }^{208} \mathrm{~Pb}$ disagree with experiment in that no strong $1^{+}$state has been found. The theoretical spin-flip strength is insufficiently fragmented. In order to explain the experimental results one has to develop theoretical models which include correlations beyond the 1p1h RPA level.

A very sophisticated RPA model which includes $2 \mathrm{p} 2 \mathrm{~h}$ as well as $\Delta \mathrm{h}$ configurations has been worked out by Cha et al. ${ }^{11}$ and applied to ${ }^{90} \mathrm{Zr}$ and ${ }^{208} \mathrm{~Pb}$. These authors show that the inclusion of $2 \mathrm{p} 2 \mathrm{~h}$ correlations (in RPA) reduces the $M 1$ strength in ${ }^{208} \mathrm{~Pb}$, below $11 \mathrm{MeV}$ by more than $30 \%$ and admixture of $\Delta \mathrm{h}$ components gives a further quenching of about $20 \%$. The total theoretical strength is in good agreement with the most recent experiment, as shown in Table III. However, this model not only predicts the total $B(M 1)$ value correctly but it also gives an $M 1$ strength distribution very close to what is measured. The results are compared in Fig. 10.

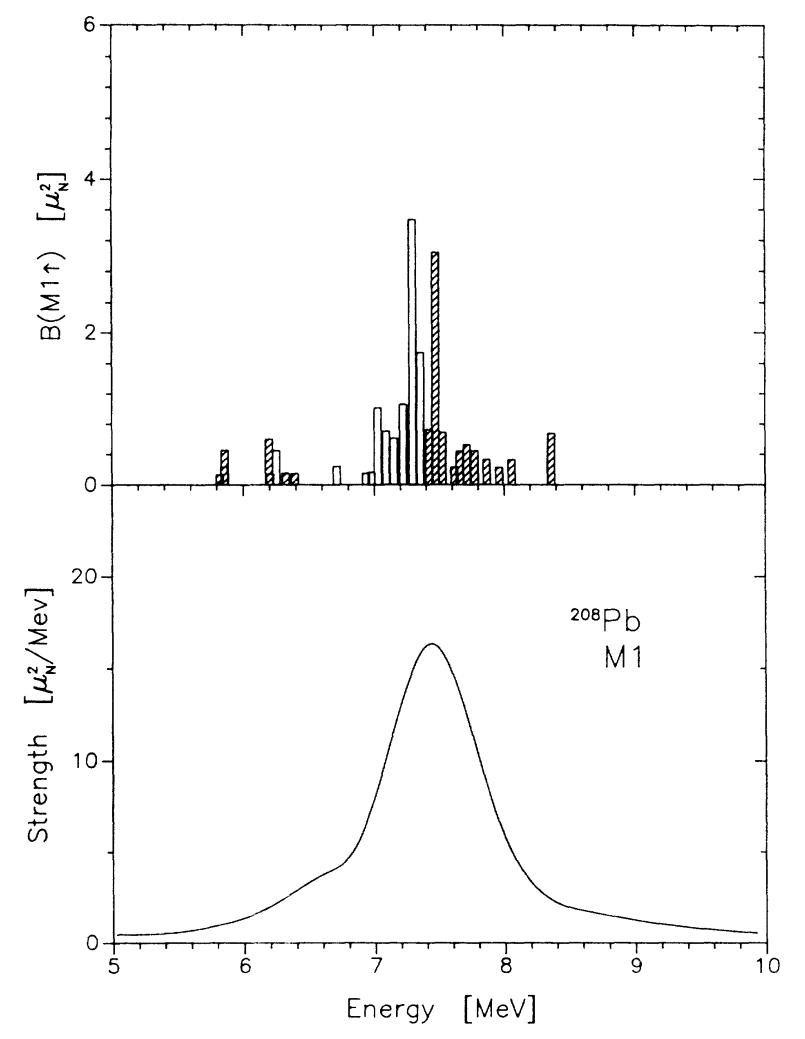

FIG. 10. In the upper part the experimentally detected $B(M 1)$ strength in ${ }^{208} \mathrm{~Pb}$ is shown (Ref. 4). The open histogram indicates the results by Laszewski et al. (Ref. 4) obtained with polarized photons (below neutron threshold) and the shaded histogram the known $B(M 1)$ strength above threshold (Ref. 5). The theoretical calculation shown in the lower part has been performed by Cha et al., (Ref. 11) within the $1 \mathrm{p} 1 \mathrm{~h}+2 \mathrm{p} 2 \mathrm{~h}$ RPA also including $1 \Delta 1 \mathrm{~h}$ correlations. The total theoretical strength below $11 \mathrm{MeV}$ is $B(M 1) \uparrow=20.4 \mu_{N}^{2}$, which should be compared with the total experimental strength below $8.5 \mathrm{MeV}$ of $B(M 1) \uparrow=17.5 \mu_{N}^{2}$ (Ref. 4).

TABLE III. The values given in the third and fourth columns for ${ }^{156} \mathrm{Gd}$ and ${ }^{238} \mathrm{U}$ are QRPA results summed up from 4-10 MeV and 3.5-10 MeV, respectively. The $B(M 1)$ strengths shown in the fifth column are the integrated values below $11 \mathrm{MeV}$. The results for the uncorrelated two-quasiparticle states in ${ }^{156} \mathrm{Gd}$ and ${ }^{238} \mathrm{U}$ (second column) are not given because they also include components of the spurious (isoscalar) $1^{+}$state.

\begin{tabular}{|c|c|c|c|c|c|}
\hline \multirow[b]{2}{*}{${ }^{90} \mathrm{Zr}$} & $\begin{array}{c}\text { Independent particle } \\
\text { model } \\
\text { free operator }\end{array}$ & ind $\frac{T}{1}$ & PA & $\begin{array}{l}\text { ength in } \mu_{N}^{2} \\
\begin{array}{l}1 \mathrm{p} 1 \mathrm{~h}+2 \mathrm{p} 2 \mathrm{~h} \text { RPA } \\
\text { plus } 1 \Delta 1 \mathrm{~h} \\
\text { correlations }^{\mathrm{a}}\end{array}\end{array}$ & \multirow{2}{*}{$\frac{\text { Experiment }^{\mathrm{b}}}{6.7_{-0}^{+09} 7}$} \\
\hline & 14.6 & 11.0 & 5.6 & 5.8 & \\
\hline${ }^{156} \mathrm{Gd}$ & & 28.4 & 15.8 & & \\
\hline${ }^{208} \mathrm{~Pb}$ & 45.6 & 35.6 & 19.5 & 20.4 & $17.5_{-1.7}^{+20}$ \\
\hline${ }^{238} \mathbf{U}$ & & 36.3 & 18.6 & & \\
\hline
\end{tabular}

${ }^{\mathrm{a}}$ Reference 11 .

${ }^{\mathrm{b}}$ References 3 and 4. 
In an analysis of magnetic moments and transitions Speth et al. ${ }^{19}$ have derived an effective magnetic-moment operator which accounts for effects beyond the $1 \mathrm{p} 1 \mathrm{~h}$ RPA correlations. This operator is suitable for use in the present $1 \mathrm{p} 1 \mathrm{~h}$ RPA calculations. The results for ${ }^{90} \mathrm{Zr}$ and ${ }^{208} \mathrm{~Pb}$ are given in the third row of Table III and as dashed lines in Fig. 9. The total strength calculated in 1plh RPA with the effective magnetic operator is close to what is obtained by the much more elaborate calculations of Ref. 11 (note: the effective operator parametrizes the effect of higher-order correlations; the underlying physics is discussed in Ref. 11). As we can see from Figs. 9 and 7, the effective operator does not influence the shape of the M1 strength distribution, but only its absolute magnitude. If, however, we fold a Gaussian distribution into the 1p1h RPA strength function (including the effective operator) we obtain an $M 1$ distribution very similar to that in the lower part of Fig. 10.

We now simulate the effects of $\Delta$-particle nucleon-hole and $2 \mathrm{p} 2 \mathrm{~h}$ admixtures by using effective $g_{s}$ factors and folding a Gaussian spreading-distribution width in all the levels of the 1plh QRPA $M 1$ strength functions. The theoretical distributions depend of course on the width of the Gaussian. As the number of $2 \mathrm{p} 2 \mathrm{~h}$ states increases with the excitation energy we use a Gaussian with a width that increases with energy. From the theoretical and experimental results of ${ }^{208} \mathrm{~Pb}$ in Fig. 10 one obtains a width of $\Gamma \sim 1 \mathrm{MeV}$. However, one has to bear in mind that the level density in the doubly closed shell nucleus ${ }^{208} \mathrm{~Pb}$ is low compared to similar excitation energy in deformed heavy mass nuclei. Therefore a width of the order of $\Gamma=1-2 \mathrm{MeV}$ FWHM might be a realistic value. The $M 1$ distribution of ${ }^{156} \mathrm{Gd}$ in the bottom part of Fig, 7 has been obtained by folding all the individual levels with Gaussians with widths that increase linearly from $\Gamma=0.5$ $\mathrm{MeV}$ at $E_{\mathrm{ex}}=4 \mathrm{MeV}$ to $\Gamma=2 \mathrm{MeV}$ at $E_{\mathrm{ex}}=10 \mathrm{MeV}$. For comparison the same procedure has also been applied to

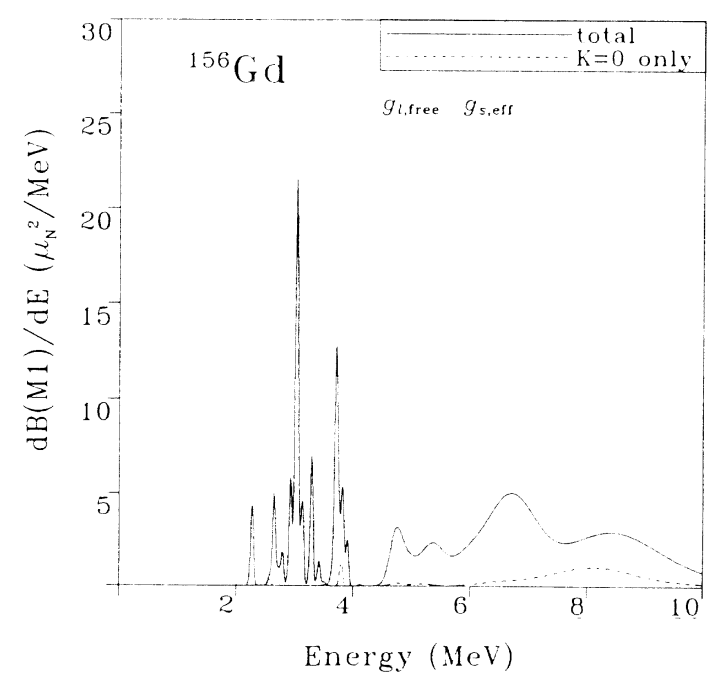

FIG. 11. The complete $B(M 1)$ spectrum of ${ }^{156} \mathrm{Gd}$ up to the excitation energy of $E_{\text {ex }}=10 \mathrm{MeV}$ calculated within the QRPA. Gaussian functions have been folded into the individual levels, the width of which increases linearly from $\Gamma=0$ at $E_{\mathrm{ex}}=4 \mathrm{MeV}$ to $\Gamma=2 \mathrm{MeV}$ at $E_{\mathrm{ex}}=10 \mathrm{MeV}$, but a minimum width of $\Gamma=60$ $\mathrm{keV}$ is used whenever $\Gamma$ would be smaller than that. the uncorrelated two-quasiparticle result, Fig. 6. The differences between the distributions in Figs. 6 and 7 (lower part, solid lines) are due to the repulsive spinisospin interaction in this channel that shifts the isovector spin-flip strength up in energy by several $\mathrm{MeV}$.

Another choice of widths for the same folding procedure has been used for the distribution shown in Fig. 11 , where the low-energy $1^{+}$spectrum which is dominated by the orbital contribution is also included: between $E_{\mathrm{ex}}=4 \mathrm{MeV}$ and $E_{\mathrm{ex}}=10 \mathrm{MeV}$ the width increases linearly from $\Gamma=0$ to $\Gamma=2 \mathrm{MeV}$; but in order to avoid too sharp spikes, a minimum width of $\Gamma=60 \mathrm{keV}$ has been used (which might correspond to a not-too-high detector resolution). Comparing the part above $4 \mathrm{MeV}$ with the corresponding curve of Fig. 7 one sees only small differences. Bearing in mind the experimental difficulties which had to be overcome to detect the (strongest) low-lying $1^{+}$states, one easily understands why so far only little is known about the high-lying $M 1$ strength.

The $M 1$ strength in ${ }^{238} \mathrm{U}$ is shifted to lower energies compared to ${ }^{156} \mathrm{Gd}$ because the single-particle spacing is proportional to $A^{-1 / 3}$. The total strength in ${ }^{238} \mathrm{U}$ in the range between 3.5 and $10 \mathrm{MeV}$ excitation energy is slightly larger than in ${ }^{156} \mathrm{Gd}$. In Fig. 12 the QRPA results for the $M 1$ distribution of ${ }^{238} \mathrm{U}$ beyond $3 \mathrm{MeV}$ are shown. The distribution is of predominantly spin-flip character and can be directly compared with the corresponding results given in the bottom part of Fig. 7. The Gaussian width was varied linearly with energy starting from $\Gamma=0.5 \mathrm{MeV}$ at $E_{\mathrm{ex}}=3.5 \mathrm{MeV}$ to $\Gamma=2 \mathrm{MeV}$ at $E_{\mathrm{ex}}=10$ $\mathrm{MeV}$. This averaging procedure yields a pronounced two-resonance structure. Finally, in Fig. 13 we give also the complete spectrum of ${ }^{238} \mathrm{U}$. Again a minimum width of $\Gamma=60 \mathrm{keV}$ is employed, and a linear increase from $\Gamma=0$ at $3 \mathrm{MeV}$ to $\Gamma=2 \mathrm{MeV}$ at $E_{\mathrm{ex}}=9 \mathrm{MeV}$ is assumed.

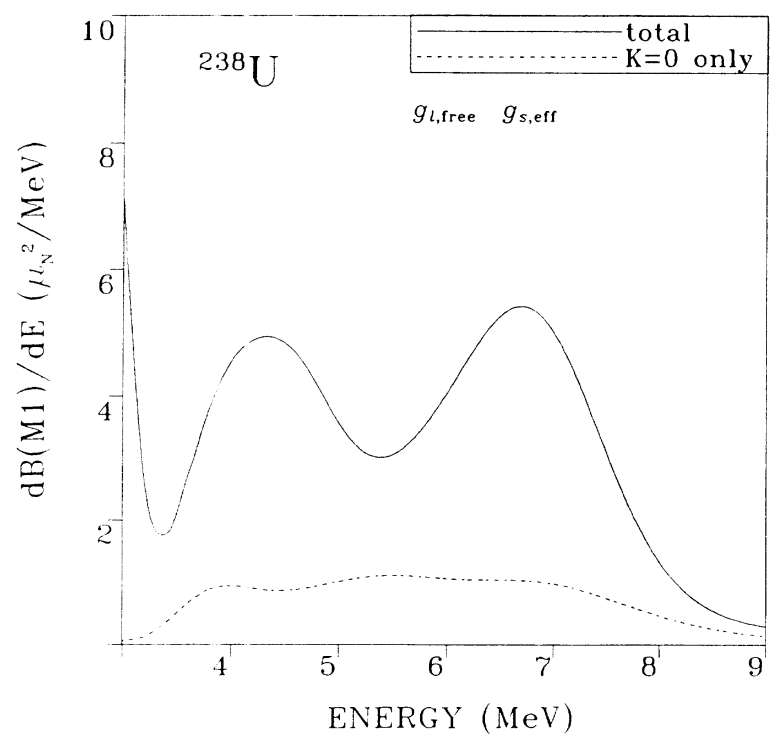

FIG. 12. $B(M 1)$ strength distribution in ${ }^{238} \mathrm{U}$ calculated within the QRPA. The Gaussian width increases linearly from $\Gamma=0.5 \mathrm{MeV}$ at $E_{\mathrm{ex}}=3.5 \mathrm{MeV}$ to $\Gamma=2 \mathrm{MeV}$ at $E_{\mathrm{ex}}=9 \mathrm{MeV}$. 


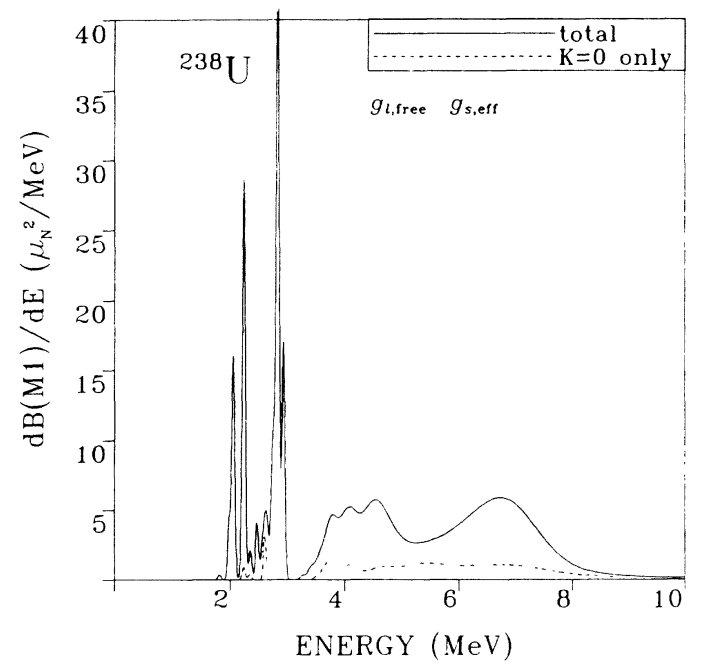

FIG. 13. The complete $B(M 1)$ spectrum of ${ }^{238} \mathrm{U}$ up to the excitation energy of $E_{\mathrm{ex}}=10 \mathrm{MeV}$ derived within the QRPA. Similar to Fig. 11 a minimum width of $\Gamma=60 \mathrm{keV}$ has been employed, and a linear increase from $\Gamma=0$ at $E_{\mathrm{ex}}=3.5 \mathrm{MeV}$ to $\Gamma=2 \mathrm{MeV}$ at $E_{\mathrm{ex}}=9 \mathrm{MeV}$. Note: The structure of the spectrum below $E_{\mathrm{ex}}=3.5 \mathrm{MeV}$ depends sensitively on the energies of the individual single-particle states which are not known with the necessary precision. Therefore we can only predict the total $B(M 1)$ strength below $E_{\mathrm{ex}}=3.5 \mathrm{MeV}$ with confidence.

Here we point out that the detailed level structure below 3.5 MeV depends sensitively on the energies of the individual single-particle states around the Fermi surface, which are not known with the necessary degree of precision. We expect, however, that the total theoretical $M 1$ strength below $3.5 \mathrm{MeV}$ is reasonably reliable.

We mentioned in Sec. II that in spheroidal nuclei only the projection $K$ of the total angular momentum of the intrinsic wave function on the symmetry axis is a good quantum number. Therefore we expect $K^{\pi}=1^{+}$and $K^{\pi}=0^{+}$contributions to the spin-flip dipole strength distribution. It is well known that in the case of the electric dipole resonance the $E 1$ distribution splits into $K^{\pi}=1^{-}$ and $K^{\pi}=0^{-}$parts which are well separated from each other. This is not the case for the $1^{+}$states. The $K^{\pi}=0^{+}$part is given separately as dashed lines in Figs. 11,13 . If we compare the $K^{\pi}=0^{+}$part with the sum of the $K^{\pi}=0^{+}$and $1^{+}$contributions (solid line) in the QRPA results of ${ }^{238} \mathrm{U}$, then one observes that the $K^{\pi}=0^{+}$contribution is only a smooth background. In
${ }^{156} \mathrm{Gd}$ (upper and middle parts of Fig. 11) the situation is slightly different. The main $K^{\pi}=0^{+}$part is close to the minimum of two well-separated $K^{\pi}=1^{+}$contributions. Therefore we do not obtain in ${ }^{156} \mathrm{Gd}$ a double resonance structure as we do in ${ }^{238} \mathrm{U}$.

We can summarize the results on the spin-flip magnetic dipole strength as follows.

(1) We obtain from QRPA calculations appreciable $\Delta T_{Z}=0$ spin-flip dipole strength in deformed heavy mass nuclei. The total predicted strength in ${ }^{156} \mathrm{Gd}$ is intermediate between the strength in ${ }^{90} \mathrm{Zr}$ and ${ }^{208} \mathrm{~Pb}$. The calculated strength in ${ }^{238} \mathrm{U}$ is similar to the observed (and calculated) strength in ${ }^{208} \mathrm{~Pb}$.

(2) Because of deformation the strength in ${ }^{156} \mathrm{Gd}$ and ${ }^{238} \mathrm{U}$ is much more fragmented than in ${ }^{208} \mathrm{~Pb}$. It is nearly uniformly distributed (with some little structure, depending on the width of the Gaussian smearing function) between 6-9 MeV in ${ }^{156} \mathrm{Gd}$ and $4-7.5 \mathrm{MeV}$ in ${ }^{238} \mathrm{U}$.

(3) There is no pronounced splitting between the $K^{\pi}=0^{+}$and $K^{\pi}=1^{+}$components of the $M 1$ resonance in either ${ }^{156} \mathrm{Gd}$ or in ${ }^{238} \mathrm{U}$.

(4) The $\Delta T_{z}=0 M 1$ excitation is less collective than the $\Delta T_{z}=-1$ or GT excitation. The p-h interaction shifts the main $\Delta T_{z}=0$ strength $3-4 \mathrm{MeV}$ above its unperturbed position. The corresponding shift in the $\Delta T_{z}=-1 \mathrm{GT}$ excitation is about $10 \mathrm{MeV}$. At the same time, the shifted GT strength is concentrated in a smaller energy region. This variation with $\Delta T_{z}$ of the collectivity of $M 1$ excitations is by now well established. The $\Delta T_{z}=-1((p, n))$ excitations is strongly collective; the $\Delta_{T_{z}}=0\left(\left(p, p^{\prime}\right)\right.$ or $\left.\left(e^{\prime}, e\right)\right)$ excitation is weakly collective; the $\Delta T_{z}=+1((n, p))$ excitation is not collective at all.

Note added in proof. A recent $\left(p, p^{\prime}\right)$ experiment ${ }^{25}$ reports indications of the $\Delta T_{z}=0 M 1$ strength in three rare-earth nuclei. The strength-distribution appears to be double-humped which would suggest a smearing-width about half that used in Fig. 7.

\section{ACKNOWLEDGMENTS}

We would like to thank G. E. Brown and J. Wambach for valuable discussions. This work was supported in part by DOE Contract No. DE-FG02-87ER40365, by NATO Grant No. RG85/0093, and by a Humbolt Award.
${ }^{1}$ C. D. Goodman et al., Phys. Rev. Lett. 44, 1755b (1980); D. B. Bainum et al., ibid. 44, 1751 (1980).

${ }^{2}$ D. Bohle et al., Phys. Lett. 137B, 27 (1984).

${ }^{3}$ R. M. Laszewski et al., Phys. Rev. Lett. 59, 431 (1987).

${ }^{4}$ R. M. Laszewski et al., Phys. Rev. Lett. 61, 1710 (1988).

${ }^{5}$ M. Haji-Saeid et al., Phys. Rev. Lett. 45, 880 (1980).

${ }^{6} \mathrm{~W}$. Steffen et al., Phys. Lett. 95B, 23 (1980).

${ }^{7}$ D. Meuer et al., Nucl. Phys. A349, 309 (1980).

${ }^{8}$ K. Wienhard et al., Phys. Rev. Lett. 49, 18 (1982).

${ }^{9}$ N. Anantaraman et al., Phys. Rev. Lett. 46, 1318 (1981).

${ }^{10}$ S. Raman et al., Phys. Rev. Lett. 39, 598 (1977); R. Kohler et al., Phys. Rev. C 35, 1646 (1987).

${ }^{11}$ D. Cha, B. Schwesinger, J. Wambach, and J. Speth, Nucl. Phys. A430, 321 (1984).

${ }^{12}$ C. Wesselborg et al., Phys. Lett. B 207, 22 (1988).

${ }^{13}$ J. Speth and D. Zawischa, Phys. Lett. B 211, 247 (1988); 219, 529(E) (1989).

${ }^{14}$ K.-G. Dietrich et al., Phys. Lett. B 220, 351 (1989).

${ }^{15}$ D. Zawischa, J. Speth, and D. Pal, Nucl. Phys. A311, 495 (1978).

${ }^{16}$ I. Hamamoto and S. Åberg, Phys. Lett. 145B, 163 (1984).

${ }^{17}$ R. Nojarov and A. Faessler, Nucl. Phys. A484, 1 (1988). 
${ }^{18}$ A. B. Migdal, Theory of Finite Fermi Systems (Interscience, New York, 1967).

${ }^{19}$ J. Speth, E. Werner, and W. Wild, Phys. Rep. 33C, 127 (1977).

${ }^{20}$ C. D. Goodman, Progress in Particle and Nuclear Physics (Pergamon, New York), Vol. 11.

${ }^{21}$ S. Krewald, F. Osterfeld, J. Speth, and G. E. Brown, Phys. Rev. Lett. 46, 103 (1981).
${ }^{22}$ G. E. Brown and S. Raman, Comments Nucl. Part. Phys. 9, 79 (1980).

${ }^{23}$ C. D. Goodman, private communication.

${ }^{24}$ S. P. Kamerdzhiev and V. N. Tkachev, Phys. Lett. 142B, 225 (1984); Z. Phys. A 334, 19 (1989).

${ }^{25}$ D. Frekers et al., TRIUMF report, 1990 (unpublished). 